\title{
Efficacy and Safety of GPR119 Agonist DS-8500a in Japanese Patients with Type 2 Diabetes: a Randomized, Double-Blind, Placebo-Controlled, 12-Week Study
}

\author{
Yuichiro Yamada - Yasuo Terauchi · Hirotaka Watada - Yasuhiko Nakatsuka • \\ Kazuhito Shiosakai · Takuo Washio · Takashi Taguchi
}

Received: November 29, 2017 / Published online: February 27, 2018

(C) The Author(s) 2018. This article is an open access publication

\section{ABSTRACT}

Introduction: $\mathrm{G}$ protein-coupled receptor 119 (GPR119) is a promising target for the treatment of type 2 diabetes mellitus (T2DM), as both insulin and glucagon-like peptide-1 secretion can be promoted with a single drug. We compared the efficacy and safety of the GPR119 agonist DS-8500a with placebo and sitagliptin $50 \mathrm{mg}$ in Japanese patients with T2DM.

Methods: This randomized, double-blind, parallel-group comparison study was conducted in

Enhanced content To view enhanced content for this article go to https://doi.org/10.6084/m9.figshare. 5813769 .

Electronic supplementary material The online version of this article (https://doi.org/10.1007/s12325018-0668-2) contains supplementary material, which is available to authorized users.

Y. Yamada

Department of Endocrinology, Diabetes and Geriatric Medicine, Akita University Graduate School of Medicine, 1-1-1 Hondo, Akita 010-8543, Japan

Y. Terauchi

Department of Endocrinology and Metabolism, Yokohama City University Graduate School of Medicine, 3-9 Fukuura, Kanazawa-ku, Yokohama, Kanagawa 236-0004, Japan

H. Watada

Department of Metabolism \& Endocrinology, Juntendo University Graduate School of Medicine, 2-1-1 Hongo, Bunkyo-ku, Tokyo 113-8421, Japan
Japan (trial registration NCT02628392, JapicCTI-153068). Eligible patients aged $\geq 20$ years with T2DM and hemoglobin A1c $(\mathrm{HbA} 1 \mathrm{c}) \geq 7.0 \%$ and $<10.0 \%$ were randomized to receive placebo, DS-8500a $(25,50$, or $75 \mathrm{mg})$, or sitagliptin $50 \mathrm{mg}$ once daily for 12 weeks. The primary efficacy endpoint was change in HbA1c from baseline to week 12. Secondary endpoints included change in fasting plasma glucose (FPG), glucose $\mathrm{AUC}_{0-3 \mathrm{~h}}$ during a meal tolerance test, 2-hour postprandial glucose (2hr-PPG), and changes in lipid parameters (total, low-density lipoprotein (LDL-) and high-density lipoprotein (HDL-) cholesterol, and triglycerides) at week 12. Safety endpoints included adverse events, hypoglycemia, and clinical/laboratory variables.

Results: DS-8500a demonstrated dose-dependent HbA1c lowering compared with placebo at

Y. Nakatsuka · T. Taguchi $(\bowtie)$

Clinical Development Department, Daiichi Sankyo Co., Ltd., 1-2-58 Hiromachi, Shinagawa-ku, Tokyo 140-8710, Japan

e-mail: taguchi.takashi.ft@daiichisankyo.co.jp

K. Shiosakai

Biostatistics \& Data Management, Daiichi Sankyo Co., Ltd., 1-2-58 Hiromachi, Shinagawa-ku, Tokyo 140-8710, Japan

T. Washio

Asia Development Department, Daiichi Sankyo Co., Ltd., 1-2-58 Hiromachi, Shinagawa-ku, Tokyo 1408710, Japan 
week 12: change from baseline $-0.23 \%$ $(p=0.0173), \quad-0.37 \% \quad(p=0.0001), \quad$ and $-0.44 \%(p<0.0001)$ in the $25-\mathrm{mg}, 50-\mathrm{mg}$, and $75-\mathrm{mg}$ groups, respectively. At 50- and 75-mg doses, DS-8500a significantly lowered FPG, glucose $\mathrm{AUC}_{0-3 \mathrm{~h}}$, and $2 \mathrm{hr}-\mathrm{PPG}$ compared with placebo. The glucose-lowering effect was maintained up to 12 weeks. DS-8500a did not lower any of the above parameters to a greater extent than sitagliptin. Compared with placebo and sitagliptin, DS-8500a 50 and $75 \mathrm{mg}$ significantly reduced total cholesterol, LDL-cholesterol, and triglycerides, and significantly increased HDLcholesterol. All DS-8500a doses were well tolerated. Two cases of clinically relevant drug-related hypoglycemia occurred in the DS-8500a 50-mg group.

Conclusion: DS-8500a was well tolerated and demonstrated significant glucose-lowering effects and favorable changes in lipid profiles up to 12 weeks in Japanese patients with T2DM.

Funding: Daiichi Sankyo Co. Ltd.

Keywords: Efficacy; Fasting plasma glucose; G protein-coupled receptor 119; HbA1c; Japanese; Safety; Type 2 diabetes

\section{INTRODUCTION}

Type 2 diabetes mellitus (T2DM) is a chronic metabolic disorder characterized by insulin resistance and a relative lack of insulin [1]. It is also a risk factor for other serious conditions, such as neuropathy, nephropathy, arteriosclerosis, and vascular events, including ischemic heart disease, stroke, and peripheral artery disease [1]. The number of diabetic patients worldwide is increasing, which poses a serious challenge to public health $[2,3]$. If the current trend continues, it is estimated that this number will surpass 700 million patients by 2025 [4]. It is therefore critical to treat the disease early and with the appropriate treatment regimen. Management of T2DM includes diet and regular exercise; however, some people are unable to achieve target plasma glucose levels with this approach alone. Therefore, diabetes medication and insulin therapy may be needed [1].
Currently, there are several oral antidiabetic drugs available, including biguanides (e.g., metformin) and thiazolidinediones to improve insulin sensitivity [5]; sulfonylureas to aid insulin secretion [5]; dipeptidyl peptidase-4 inhibitors to reduce plasma glucose levels [5]; $\alpha$ glucosidase inhibitors to suppress or delay intestinal absorption [5]; and most recently, sodium-glucose co-transporter 2 inhibitors to enhance glucose excretion in urine [5]. Diabetic patients who have poor glycemic control through monotherapy can be treated with a combination of these agents that differ in their mechanisms of action [5].

As pancreatic $\beta$-cell dysfunction is a major contributor to T2DM progression, drugs with a mechanism of action that helps insulin secretion in pancreatic $\beta$-cells may offer a new therapeutic approach for T2DM. The G proteincoupled receptor 119 (GPR119), which has been shown to be highly expressed in the human gastrointestinal tract (small intestinal L-cells) and pancreas ( $\beta$-cells), has been identified as a novel target for antidiabetic drugs $[6,7]$. On the basis of the results of several in vitro studies, it is expected that GPR119 agonists will reduce blood glucose levels by promoting insulin secretion in a glucose-dependent manner and improve pancreatic $\beta$-cell function over longterm treatment [7-9].

DS-8500a is a novel GPR119 agonist developed by Daiichi Sankyo Co., Ltd. In non-clinical pharmacology studies, DS-8500a exerted agonistic activity in Chinese hamster ovary-K1 cells expressing human GPR119 and reversed glucose tolerance in Zucker fatty rats in an animal model of glucose intolerance [10]. A phase 1 study of DS-8500a performed in healthy Japanese men reported that a single oral dose of DS8500 a capsules up to $600 \mathrm{mg}$ and 7-day multiple oral doses of up to $300 \mathrm{mg} /$ day were tolerated by the subjects [11]. In another phase 1 study, 7-day multiple oral doses of DS-8500a tablets up to $100 \mathrm{mg} /$ day were tolerated by healthy subjects [11]. Results from a phase $2 \mathrm{a}$ study in T2DM patients evaluating DS-8500a administered at 10 or $75 \mathrm{mg}$ for 4 weeks showed a significant decrease in 24-h weighted mean glucose levels in both the DS-8500a 10-mg and 75-mg groups compared with the placebo group 
( -13.3 and $-18.9 \mathrm{mg} / \mathrm{dL}$, respectively). DS8500 a was well tolerated at both doses with no reports of serious adverse events (SAEs), clinically significant AEs, or AEs that led to treatment discontinuation [12].

On the basis of the above findings, we conducted a phase $2 \mathrm{~b}$, double-blind, parallel-group study in Japanese T2DM patients. The aim of this study was to evaluate the efficacy, safety, and dose of DS-8500a compared with placebo in patients with T2DM after a 12-week oral administration of DS-8500a at 25, 50, or $75 \mathrm{mg}$. We also investigated the efficacy of DS-8500a, relative to the active comparator sitagliptin $50 \mathrm{mg}$.

\section{METHODS}

\section{Study Design}

This was a phase $2 \mathrm{~b}$, randomized, placebo-controlled, active-controlled, double-blind, 5-parallel-group study conducted in T2DM patients to evaluate efficacy and safety of DS-8500a 25, 50 , and $75 \mathrm{mg}$ administered orally for 12 weeks (Fig. 1). This study was conducted at 25 sites in Japan (Supplementary Table S1). Subjects who received antidiabetic drugs within 90 days prior to initiation underwent a 28-day washout period (observation period 1). This was followed by observation period 2 comprising a 2 -week washout period for all subjects (those who did and did not receive any antidiabetic drugs within 90 days prior to initiation). Subjects were registered during observation period 3 prior to randomization. A randomization schedule was prepared by an independent biostatistician using hemoglobin A1c (HbA1c) at observation period $2(\leq 8.0 \%$ or $>8.0 \%)$ as a stratification factor (permuted block method). Subjects were randomized in a ratio of $1: 1: 1: 1: 1$ to the DS8500 a $25 / 50 / 75-\mathrm{mg}$, sitagliptin $50-\mathrm{mg}$, or placebo groups according to the schedule. Blinding was maintained by the independent biostatistician and those involved in the assignment, packaging, and quality assurance of study drugs.

This study was conducted in accordance with the ethical principles of the Declaration of Helsinki, Pharmaceutical Affairs Law, Good Clinical Practice, and associated Japanese regulations. The study protocol was approved by the institutional review board at each site and all patients provided written informed consent.

\section{Patients}

Eligible subjects for this study were Japanese patients with T2DM and $\geq 20$ years of age at the time of informed consent, with $\mathrm{HbA} 1 \mathrm{c} \geq 7.0 \%$

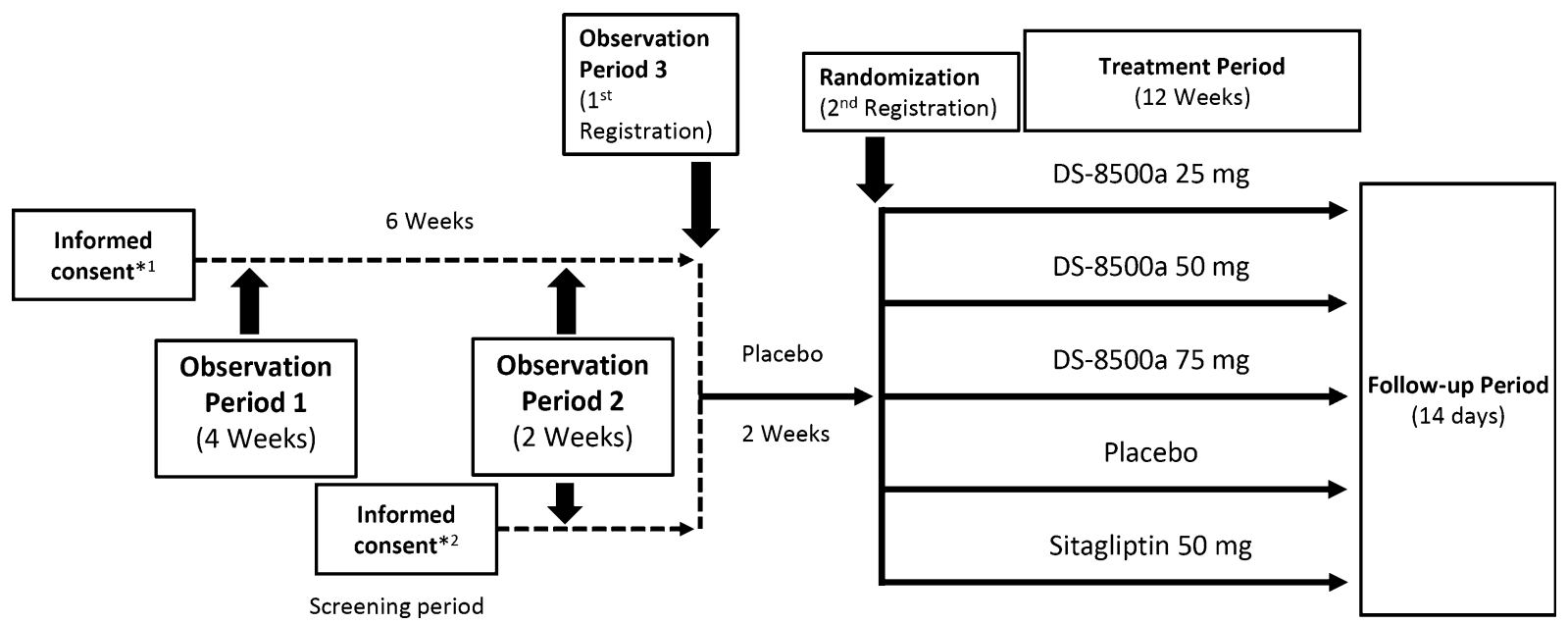

Fig. 1 Study design. ${ }^{*}$ Subjects who received a single antidiabetic drug within 90 days prior to the initiation of the observation period: 6 weeks. ${ }^{* 2}$ Subjects who had not

received an antidiabetic drug within 90 days prior to the initiation of the observation period: 2 weeks 
and $<10.0 \%$ during observation period 2 . Patients were eligible regardless of whether they had received antidiabetic drugs within 90 days prior to enrolment. The exclusion criteria included type 1 DM or a history of diabetic coma, precoma, or ketoacidosis; clinically significant diabetic retinopathy, nephropathy, or neuropathy; insulin therapy; thiazolidinedione therapy within 90 days before the initiation of the observation period; poorly controlled blood pressure [sustained systolic $(\geq 160 \mathrm{mmHg})$ or diastolic ( $\geq 100 \mathrm{mmHg}$ ) hypertension]; and body mass index (BMI) of $<18.5$ or $\geq 35.0 \mathrm{~kg} / \mathrm{m}^{2}$.

\section{Treatment}

During observation period 3, each subject was administered three placebo tablets and one placebo capsule to be taken orally once daily after breakfast for 14 days. During the treatment period, the study drugs (DS-8500a 25, 50, or $75 \mathrm{mg}$; sitagliptin $50 \mathrm{mg}$; or placebo) were administered orally after breakfast for 12 weeks. Concomitant drugs and therapies prohibited during the study included antidiabetic drugs, anorexiants, drugs that could affect the pharmacodynamics evaluation, drugs that might cause drug-drug integrations, and other study drugs. The complete list of restricted and prohibited concomitant drugs is found in the Supplementary Information (Table S2).

\section{Efficacy and Safety Endpoints and Evaluation Criteria}

The primary efficacy endpoint was the change from baseline (day - 1) in HbA1c at week 12 . The secondary efficacy endpoints included the change from baseline in HbA1c at weeks 4 and 8 , the proportion of subjects who achieved $\mathrm{HbA1c}<7.0 \%$ at week 12 , the change from baseline to week 12 in fasting plasma glucose (FPG), 2-hour postprandial plasma glucose (2hrPPG), and pharmacodynamic parameters derived from plasma glucose. Other secondary efficacy endpoints were changes from baseline at each post-dose time point in serum insulin, proinsulin, C-peptide, and 1,5-anhydroglucitol; pharmacodynamic parameters derived from serum insulin, C-peptide, peptide YY (PYY), total glucagon-like peptide-1 (GLP-1), active GLP-1, total glucose-dependent insulinotropic polypeptide (GIP), and glucagon; and total cholesterol, high-density lipoprotein (HDL-) and low-density lipoprotein (LDL-) cholesterol, and triglycerides (TGs).

AEs and safety outcomes including laboratory values, electrocardiography, body weight, and vital signs were recorded throughout the study. All hypoglycemia events were defined as clinically relevant drug-related hypoglycemia in accordance with the Guideline for Clinical Evaluation of Oral Hypoglycemic Agents (Notification No. 0709-1 [9 Jul 2010] by the Evaluation and Licensing Division, Pharmaceutical and Medical Safety Bureau, Ministry of Health, Labour and Welfare). Asymptomatic hypoglycemia was defined as a blood glucose level of $\leq 70 \mathrm{mg} / \mathrm{dL}$ without any typical hypoglycemic symptoms. Such asymptomatic events correspond to level 1 hypoglycemia according to the definition provided by the American Diabetes Association (ADA) and the International Hypoglycaemia Study Group [13].

\section{Statistical Methods}

The sample size was determined on the basis of a pairwise comparison between DS-8500a at each dose group vs placebo, based on "On Release of the Guideline for Clinical Evaluation of Oral Hypoglycemic Agents" [14] and the primary endpoint, change from baseline in HbA1c at week 12. As there are no available data on change in $\mathrm{HbA} 1 \mathrm{c}$ at week 12 after administration of DS-8500a, the sample size was selected in reference to the results of a previous study investigating the change in 24-h weighted mean glucose level at week 4 [12], and the results of a clinical study [15] investigating HbA1c after administration of sitagliptin. It was assumed that the changes in $\mathrm{HbA1c}$ at week 12 in the placebo, DS-8500a 25-mg, 50-mg, and $75-\mathrm{mg}$ groups were $0.0 \%,-0.6 \%,-0.9 \%$, $-1.0 \%$, respectively, and that the standard deviation in each treatment group was $1.0 \%$. Accordingly, a sample size of 60 patients per group was required to detect the superiority of DS-8500a at a statistical power of $90 \%$ at a two-sided significance 
level of $5 \%$ in a pairwise comparison between the placebo group and each DS-8500a group. To account for a dropout rate of approximately 10\%, a sample size of 67 patients per group (335 patients in total) was planned. The efficacy analysis was performed in the full analysis set (FAS), and the sensitivity analysis was performed in the per protocol set (PPS).

The primary endpoint was analyzed using an analysis of covariance (ANCOVA) model, with the treatment group as a fixed effect and HbA1c at baseline and presence/absence of diabetes drug use within 90 days before informed consent as covariates, and compared between each DS-8500a group and the placebo group. A fixedsequence testing approach from high dose to low dose was used to adjust for multiplicity of comparisons. A comparison between each DS8500 a group and the sitagliptin group was performed in a similar manner using an ANCOVA model, using the treatment group as a fixed effect and HbA1c at baseline as a covariate. For each comparison, the $95 \%$ confidence intervals and $p$ values were calculated with a significance level of 5\% (two-sided). The secondary endpoints were summarized for each treatment group at each time point. The safety analysis was performed in the safety analysis set, and the safety endpoints were summarized for each treatment group at each time point.

Missing data for the primary endpoint (change in HbA1c at week 12) were imputed with data obtained after the initiation of study treatment in accordance with the last observation carried forward (LOCF) method. Data for week 12 imputed by LOCF were presented as "week 12 (LOCF)" to be distinguished from data for week 12 that were not imputed.

The statistical analyses were performed using SAS system release 9.2 (SAS Inc., Cary, NC, USA).

\section{RESULTS}

\section{Patients}

The disposition of patients is summarized in Fig. 2. There were 453 patients assessed for eligibility, 368 of whom were randomized. Eighty- five patients were excluded prior to randomization for the following reasons: did not meet inclusion criteria $(n=50)$; met exclusion criteria $(n=35)$.

The placebo arm enrolled 73 patients; 73,74 , and 73 patients were enrolled in the DS-8500a 25-mg, 50-mg, 75-mg groups, respectively; and 75 patients were enrolled in the sitagliptin 50-mg group. The number of patients analyzed in each group was 72 for the placebo and DS8500 a groups, and 75 for sitagliptin. The losses from each group due to withdrawal of consent, AEs, or other are shown in Fig. 2.

The baseline characteristics of the patients were similar, with no significant differences with respect to age, sex, BMI, disease duration, or prior medication use (Table 1).

\section{Efficacy}

Compared with placebo, treatment with DS$8500 \mathrm{a}$ at $25 \mathrm{mg}, 50 \mathrm{mg}$, and $75 \mathrm{mg}$ showed a statistically significant dose-dependent decrease in $\mathrm{HbA1c}$ levels from baseline at weeks 4, 8, and 12 (Fig. 3). At week 12, the change in HbA1c from baseline compared with placebo was $-0.23 \% \quad(p=0.0173), \quad-0.37 \% \quad(p=0.0001)$, and $-0.44 \% \quad(p<0.0001)$ in the DS-8500a 25-mg, 50-mg, and 75-mg groups, respectively (Fig. 3). However, compared with sitagliptin, DS-8500a did not show greater lowering effects on HbA1c (Fig. 3, Table 2).

Compared with placebo, DS-8500a $50 \mathrm{mg}$ and $75 \mathrm{mg}$ had significant lowering effects on FPG $[-12.7 \mathrm{mg} / \mathrm{dL}(p=0.0004)$ and $-14.4 \mathrm{mg} /$ $\mathrm{dL}(p<0.0001)]$ (Fig. 4$)$, as well as glucose AUC $_{0-3 \mathrm{~h}} \quad[-50.6 \mathrm{mg} / \mathrm{dL} \times \mathrm{h} \quad(p=0.002) \quad$ and $-59.7 \mathrm{mg} / \mathrm{dL} \times \mathrm{h} \quad(p=0.0003)]$, and $2 \mathrm{hr}-\mathrm{PPG}$ $[-18.5 \mathrm{mg} / \mathrm{dL}(p=0.0102)$ and $-22.0 \mathrm{mg} / \mathrm{dL}$ $(p=0.0022)$, respectively]. DS-8500a at any dose did not lower any of these parameters to a greater extent than sitagliptin (Fig. 4).

DS-8500a also affected lipid parameters. Compared with placebo, both DS-8500a at 50 and $75 \mathrm{mg}$ significantly reduced total cholesterol $[-7.0 \%(p<0.0001),-6.2 \%(p=0.0002)$, respectively vs placebo], LDL-cholesterol $[-7.3 \% \quad(p=0.0044), \quad-8.1 \% \quad(p=0.0014)$, respectively], and TGs $[-32.7 \%(p<0.0001)$, 


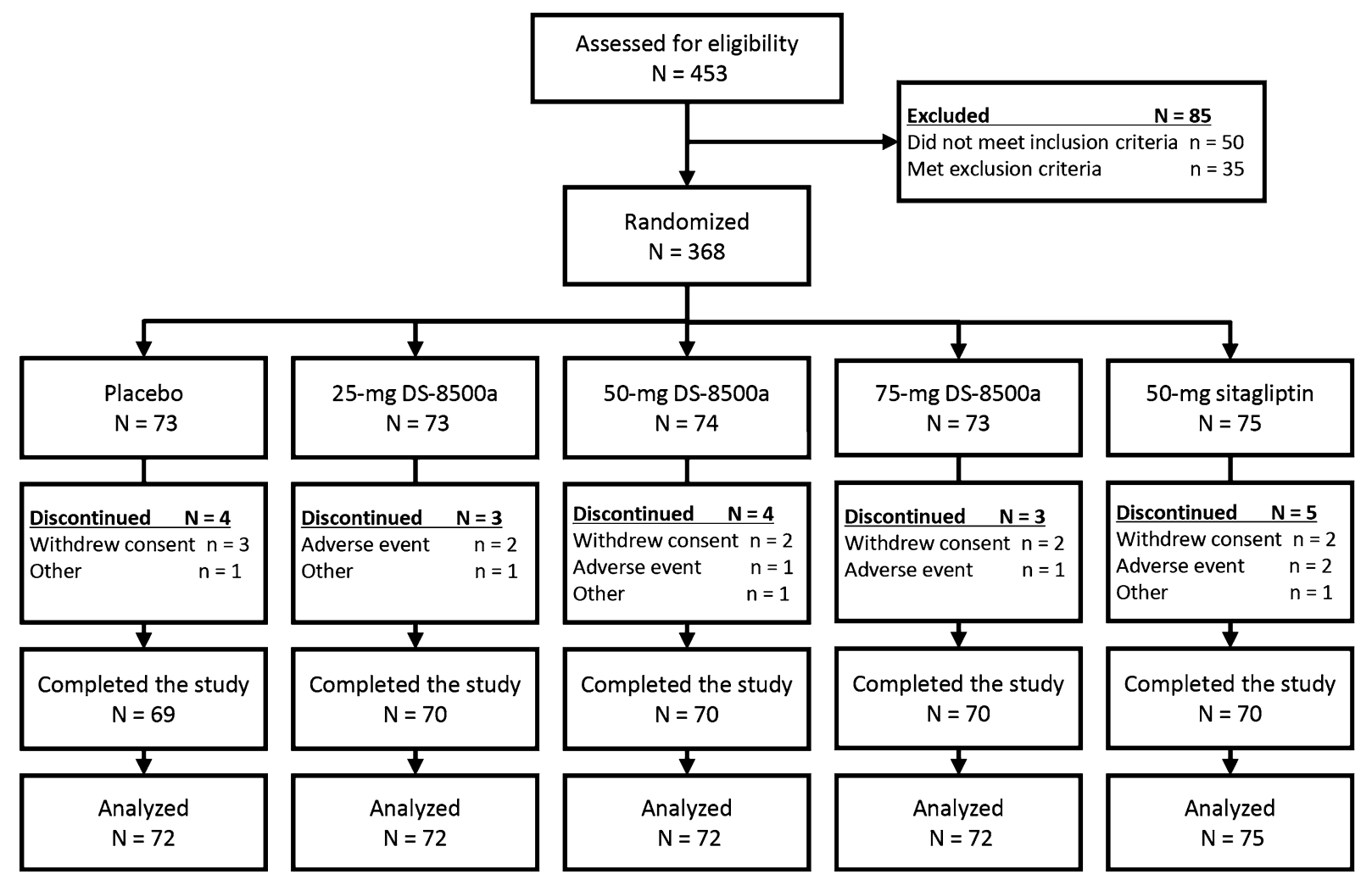

Fig. 2 Patient disposition. "Analyzed" refers to the full analysis set rather than the per protocol set

$-30.9 \%(p<0.0001)$, respectively], and significantly increased HDL-cholesterol [5.9\%, $(p=0.0032), 6.6 \%,(p=0.0009)$, respectively] (Fig. 5). Furthermore, DS-8500a at 50 and $75 \mathrm{mg}$ induced similar significant improvements in all lipid parameters compared with sitagliptin.

The changes in $\mathrm{AUC}_{0-3 \mathrm{~h}}$ from baseline in insulin, C-peptide, total GLP-1, active GLP-1, PYY, GIP, and glucagon at week 12 are shown in Table 2. In the DS-8500a groups, $\mathrm{AUC}_{0-3 \mathrm{~h}}$ for insulin and C-peptide was slightly increased compared with placebo from baseline to week 12. For PYY and GIP, there was a statistically significant increase in DS-8500a groups compared with placebo. However, for total GLP1 , active GLP-1, and glucagon, no significant difference was observed between the placebo and the DS-8500a groups.

\section{Safety}

The incidence of AEs, including serious AEs, severe AEs, and treatment-emergent AEs
(TEAEs) is presented in Table 3. Although no serious or severe TEAEs were considered to be related to the study drugs in any of the treatment arms, three serious TEAEs occurred: two in the DS-8500a 25-mg arm and one in the DS8500 a $50-\mathrm{mg}$ arm. One patient in the DS-8500a 25-mg arm was a 62-year-old man with dyslipidemia who presented with myocardial infarction 2 days after initiating treatment. He discontinued treatment and underwent emergency coronary angiography and subsequently recovered. This serious AE was considered moderate in severity and unrelated to the study drug. The second TEAE in the DS-8500a 25-mg arm involved a 43-year old man who accidentally fell and fractured his wrist approximately 2 months after initiating treatment. He underwent surgery for a suspected comminuted wrist fracture several days later. As an additional hypoglycemic drug was necessary to control his blood glucose preoperatively, he had to discontinue the study treatment. He subsequently recovered from this moderately serious $\mathrm{AE}$, 
Table 1 Baseline patient characteristics

\begin{tabular}{|c|c|c|c|c|c|c|}
\hline & $\begin{array}{l}\text { Placebo } \\
n=72\end{array}$ & $\begin{array}{l}\text { DS-8500a } \\
25 \mathrm{mg} \\
n=72\end{array}$ & $\begin{array}{l}\text { DS-8500a } \\
50 \mathrm{mg} \\
n=72\end{array}$ & $\begin{array}{l}\text { DS-8500a } \\
75 \mathrm{mg} \\
n=72\end{array}$ & $\begin{array}{l}\text { Sitagliptin } \\
\mathbf{5 0 ~} \mathbf{~ m g} \\
n=75\end{array}$ & $\begin{array}{l}\text { Total } \\
N=363\end{array}$ \\
\hline Age (years) & $61.5 \pm 8.5$ & $59.5 \pm 9.3$ & $61.4 \pm 9.5$ & $61.3 \pm 8.5$ & $61.1 \pm 9.4$ & $61.0 \pm 9.0$ \\
\hline \multicolumn{7}{|l|}{ Sex } \\
\hline Male & $57(79.2)$ & $53(73.6)$ & $51(70.8)$ & $57(79.2)$ & $61(81.3)$ & $279(76.9)$ \\
\hline Female & $15(20.8)$ & $19(26.4)$ & $21(29.2)$ & $15(20.8)$ & $14(18.7)$ & $84(23.1)$ \\
\hline BMI $\left(\mathrm{kg} / \mathrm{m}^{2}\right)$ & $25.21 \pm 3.69$ & $24.59 \pm 3.39$ & $25.19 \pm 3.73$ & $25.59 \pm 3.34$ & $24.81 \pm 3.29$ & $25.08 \pm 3.49$ \\
\hline $\begin{array}{l}\text { Duration of DM } \\
\text { (years) }\end{array}$ & $8.26 \pm 6.08$ & $6.75 \pm 4.84$ & $8.36 \pm 6.09$ & $7.57 \pm 6.33$ & $7.97 \pm 5.45$ & $7.79 \pm 5.78$ \\
\hline \multicolumn{7}{|l|}{ Prior use of OHAs } \\
\hline Absence & $30(41.7)$ & $32(44.4)$ & $27(37.5)$ & $33(45.8)$ & $30(40.0)$ & $152(41.9)$ \\
\hline Presence & $42(58.3)$ & $40(55.6)$ & $45(62.5)$ & $39(54.2)$ & $45(60.0)$ & $211(58.1)$ \\
\hline HbA1c (\%) & $7.96 \pm 0.69$ & $7.93 \pm 0.71$ & $8.07 \pm 0.88$ & $7.99 \pm 0.75$ & $8.04 \pm 0.76$ & $8.00 \pm 0.76$ \\
\hline $\mathrm{FPG}(\mathrm{mg} / \mathrm{dL})$ & $193.1 \pm 29.1$ & $193.1 \pm 29.1$ & $203.6 \pm 40.9$ & $199.6 \pm 34.6$ & $199.9 \pm 33.5$ & $199.3 \pm 34.2$ \\
\hline
\end{tabular}

Results are presented as the mean \pm standard deviation or $\mathrm{n}(\%)$

$B M I$ body mass index, $D M$ diabetes mellitus, $O H A s$ oral hypoglycemic agents, $H b A 1 c$ hemoglobin A1c, FPG fasting plasma glucose

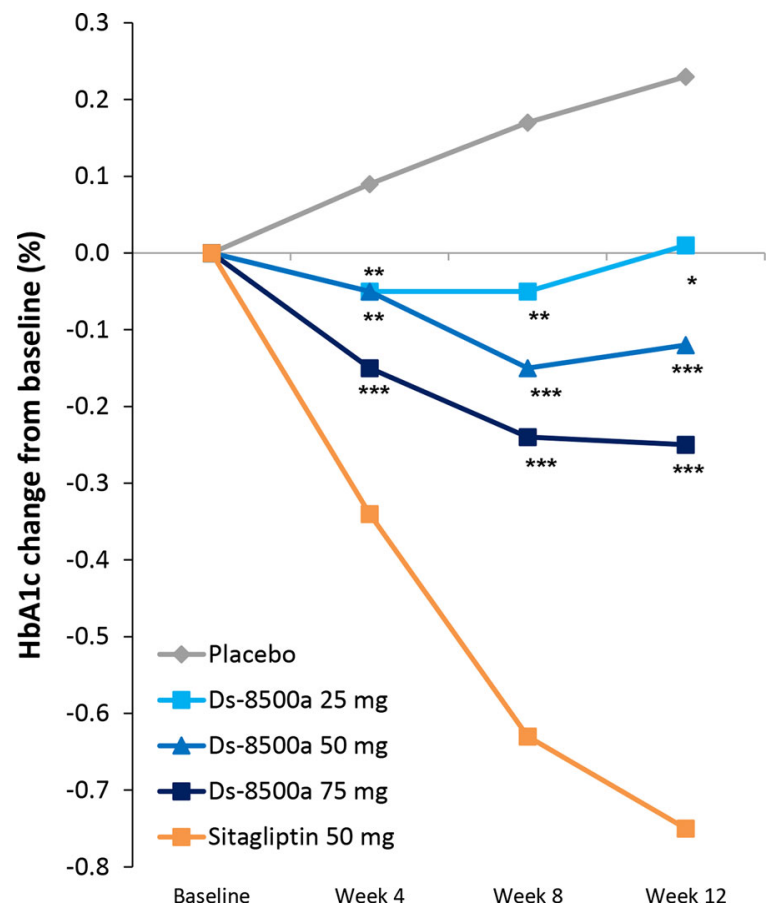

Fig. 3 Change in HbAlc from baseline to week 12 . ${ }^{*} p<0.05,{ }^{* *} p<0.01,{ }^{* * *} p<0.001$ vs placebo which was not considered related to the study drug. The patient in the DS-8500a 50-mg arm was a 50-year-old man who fell after accidentally stumbling on a step, and suffered a right orbital floor/orbital wall fracture roughly 3 months after initiating study drug treatment. This event resolved without changes in the dose of the study drug, and it was not considered related to the study drug. The two serious AEs described above in the DS-8500a 25-mg arm (myocardial infarction and wrist fracture) led to withdrawal from the study. Additionally, one event in the DS-8500a 75-mg arm led to withdrawal from the study: a 54-year-old male patient with dyslipidemia, liver function disorder, and dental caries presented with decreased hemoglobin approximately 1 month after initiating DS-8500a $75 \mathrm{mg}$. This event was considered unrelated to the study drug and was attributed to decreased dietary intake after the patient suffered an influenza infection. DS8500 a was well tolerated at all doses.

There were two cases of clinically relevant drug-related hypoglycemia reported in the DS- 


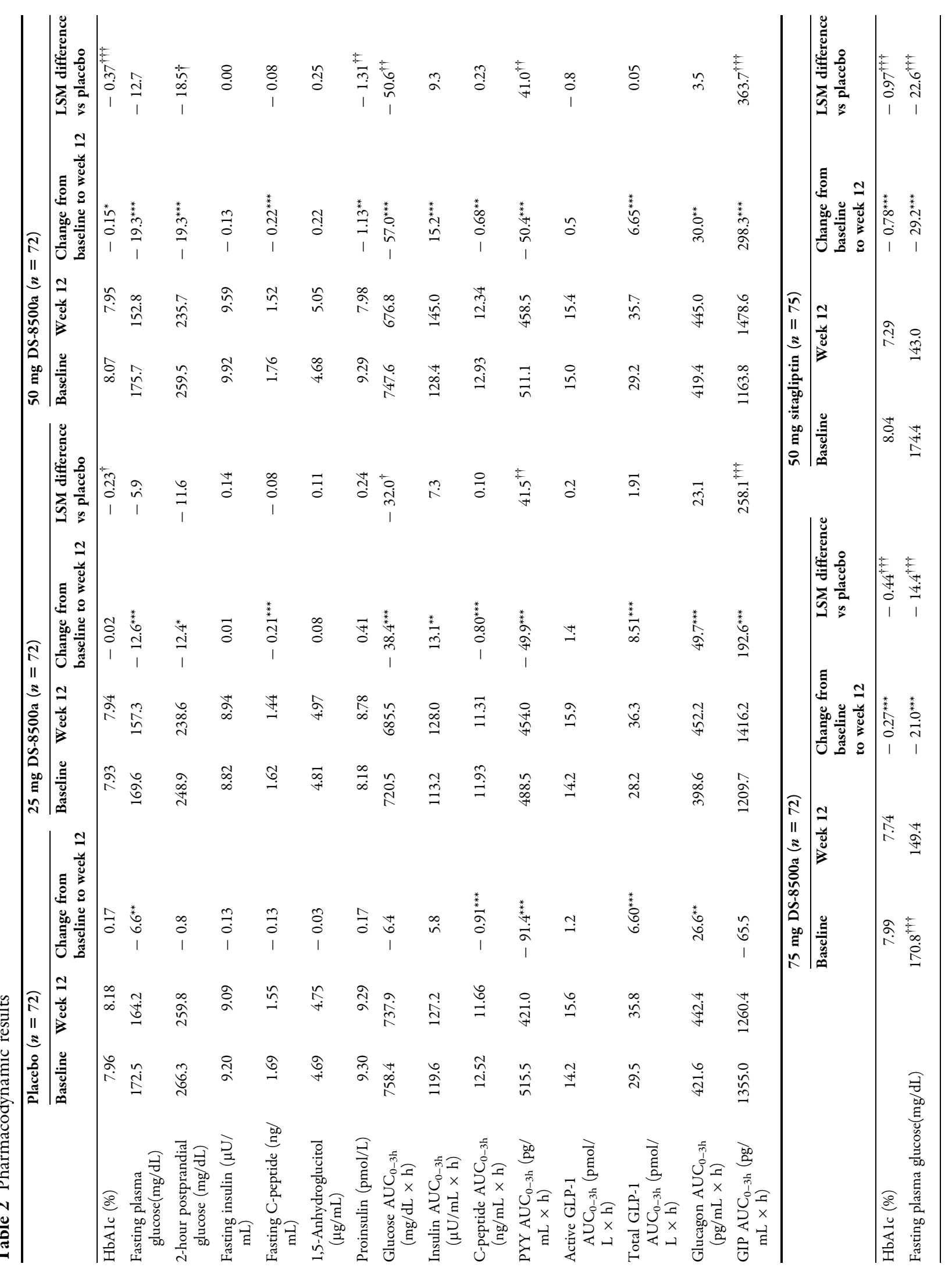




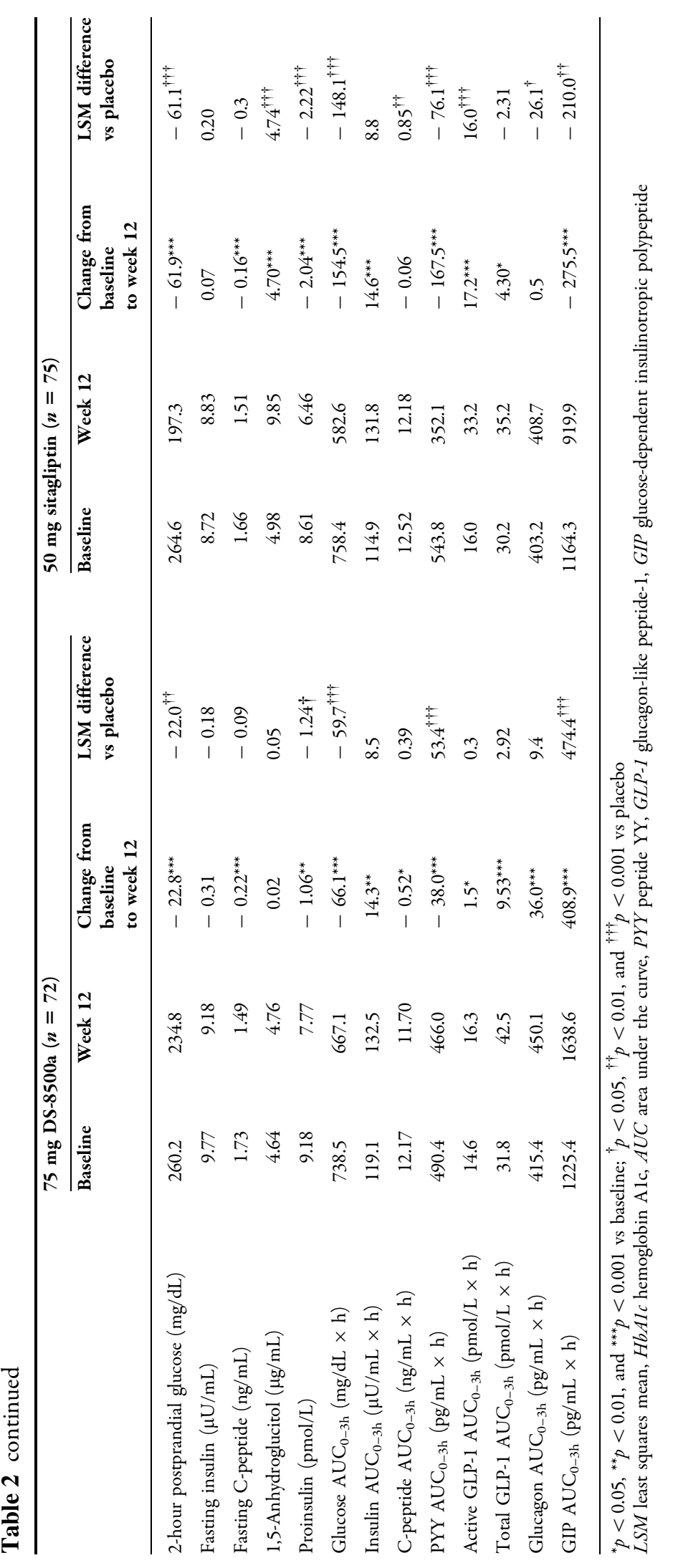




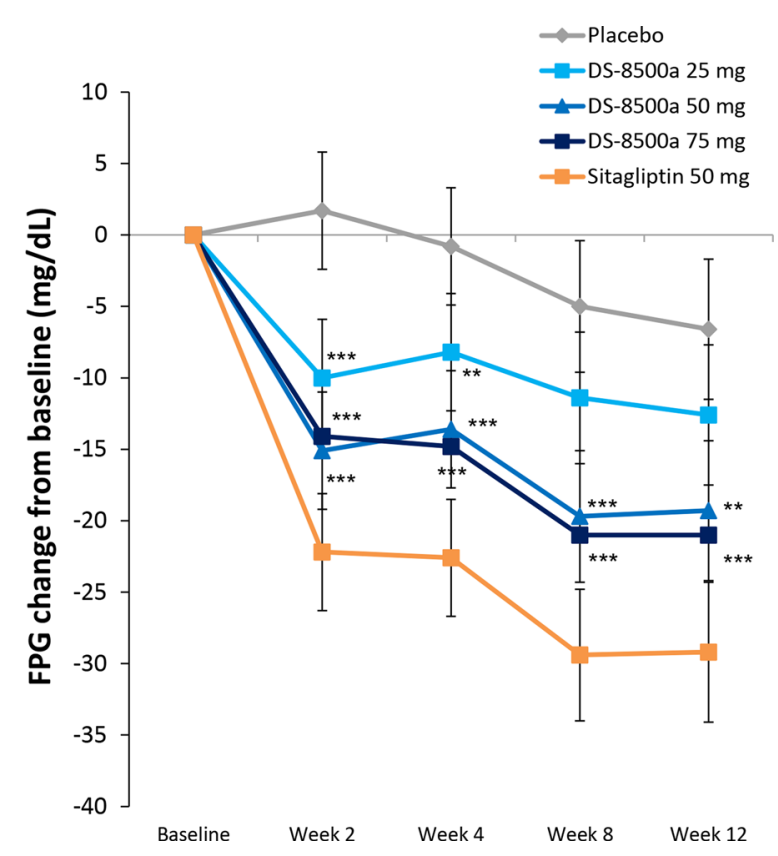

Fig. 4 Change in fasting plasma glucose (FPG) from baseline to Week $12 .{ }^{* *} p<0.01,{ }^{* * *} p<0.001$ vs placebo

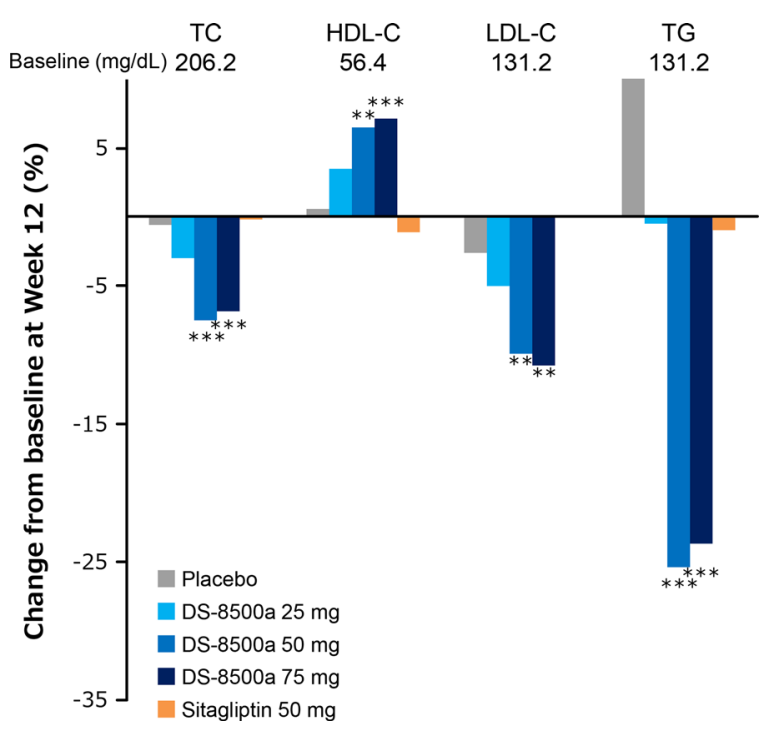

Fig. 5 Change in lipid parameters from baseline to week 12 (full analysis set). TC total cholesterol, HDL-C high-density lipoprotein cholesterol, LDL-C low-density lipoprotein cholesterol, TG triglycerides. ${ }^{*} p<0.05$, ${ }^{* *} p<0.01,{ }^{* * *} p<0.001$ vs placebo (ANCOVA)

8500a 50-mg group. These patients were asymptomatic (blood glucose level $\leq 70 \mathrm{mg} / \mathrm{dL}$ without showing any typical hypoglycemic symptoms). One of the patients was a 69-yearold man who presented with two episodes of asymptomatic hypoglycemia (glucose $=60 \mathrm{mg}$ / dL) 1 month after initiating study treatment. Both episodes resolved without treatment or any changes in the study drug dose. The other patient was a 74-year-old woman who presented with asymptomatic hypoglycemia the day after beginning treatment. Her glucose level was $60 \mathrm{mg} / \mathrm{dL}$, and she recovered without treatment or any changes in the study drug dose.

\section{DISCUSSION}

This was a phase $2 \mathrm{~b}$ study conducted in Japan to evaluate the efficacy and safety of DS-8500a (25-, 50-, 75-mg doses), a novel GPR119 agonist, in patients with T2DM. The results demonstrated that compared with placebo, DS-8500a at once-daily doses of 25,50 , or $75 \mathrm{mg}$ showed dose-dependent decreases in HbA1c levels from baseline to week 12. DS-8500a 50 and $75 \mathrm{mg}$ also demonstrated significant lowering effects on FPG, glucose $\mathrm{AUC}_{0-3 \mathrm{~h}}$, and $2 \mathrm{hr}-\mathrm{PPG}$ compared with placebo. All doses of DS-8500a were well tolerated, and there were no serious drugrelated TEAEs reported in any treatment group.

The glucose-lowering effects of other GPR119 agonists are typically abrogated after $2-4$ weeks of treatment $[16,17]$. However, in the present study, the decreases in FPG, 2hrPPG, glucose $\mathrm{AUC}_{0-3 \mathrm{~h}}$, and HbA1c by DS-8500a were maintained over time up to 12 weeks. This result can be partially explained by structural differences between the different GPR119 molecules. Other GPR119 agonists contain a piperidine ring and have both agonistic and antagonistic activities [18]. In contrast, DS$8500 a$, which does not contain a piperidine ring, has full agonist activity. Additionally, racial differences have been observed when comparing T2DM pathology between Asians and Caucasians $[19,20]$, with insulin secretagogues showing greater efficacy in Asians $[21,22]$.

In a hyperglycemic clamp study, patients treated with DS-8500a $75 \mathrm{mg}$ for 4 weeks exhibited increases in first- and second-phase 
Table 3 Adverse events

\begin{tabular}{|c|c|c|c|c|c|}
\hline & $\begin{array}{l}\text { Placebo } \\
(N=73)\end{array}$ & $\begin{array}{l}\text { DS-8500a } \\
25 \mathrm{mg}(N=73)\end{array}$ & $\begin{array}{l}\text { DS-8500a } \\
50 \mathrm{mg}(N=74)\end{array}$ & $\begin{array}{l}\text { DS-8500a } \\
75 \mathrm{mg}(N=73)\end{array}$ & $\begin{array}{l}\text { Sitagliptin } \\
50 \mathrm{mg}(N=75)\end{array}$ \\
\hline TEAE & $21(28.8)$ & $27(37.0)$ & $24(32.4)$ & $22(30.1)$ & $27(36.0)$ \\
\hline Drug-related TEAE & $2(2.7)$ & $2(2.7)$ & $4(5.4)$ & $2(2.7)$ & $5(6.7)$ \\
\hline Serious TEAE & $2(2.7)$ & $2(2.7)$ & $1(1.4)$ & $0(0.0)$ & $0(0.0)$ \\
\hline Serious drug-related TEAE & $0(0.0)$ & $0(0.0)$ & $0(0.0)$ & $0(0.0)$ & $0(0.0)$ \\
\hline Severe TEAE & $0(0.0)$ & $0(0.0)$ & $0(0.0)$ & $0(0.0)$ & $0(0.0)$ \\
\hline Severe drug-related TEAE & $0(0.0)$ & $0(0.0)$ & $0(0.0)$ & $0(0.0)$ & $0(0.0)$ \\
\hline Clinically relevant hypoglycemia & $0(0.0)$ & $0(0.0)$ & $2(2.7)$ & $0(0.0)$ & $0(0.0)$ \\
\hline $\begin{array}{l}\text { Clinically relevant drug- } \\
\text { related hypoglycemia }\end{array}$ & $0(0.0)$ & $0(0.0)$ & $2(2.7)$ & $0(0.0)$ & $0(0.0)$ \\
\hline $\begin{array}{l}\text { Clinically relevant } \\
\text { cardiovascular events }\end{array}$ & $0(0.0)$ & $1(1.4)$ & $0(0.0)$ & $0(0.0)$ & $0(0.0)$ \\
\hline $\begin{array}{l}\text { Clinically relevant drug- } \\
\text { related cardiovascular events }\end{array}$ & $0(0.0)$ & $0(0.0)$ & $0(0.0)$ & $0(0.0)$ & $0(0.0)$ \\
\hline $\begin{array}{l}\text { TEAE leading to dose } \\
\text { withdrawal }\end{array}$ & $0(0.0)$ & $2(2.7)$ & $0(0.0)$ & $1(1.4)$ & $1(1.3)$ \\
\hline
\end{tabular}

TEAE treatment-emergent adverse event

insulin secretion: AUC $(p=0.0011, p=0.0112)$, as well as first/second-phase C-peptide secretion: AUC $(p=0.0012, p<0.0001)$ compared with placebo [23]. From these results, we hypothesized that the decrease in 2hr-PPG after breakfast may have been due to increased insulin secretion, which was observed in the clamp study. However, in this study we did not observe an increase in insulin secretion vs placebo with either DS-8500a or sitagliptin treatment.

In this study, sitagliptin was chosen as a comparator because sitagliptin is moderately effective, highly safe, and is the most widely used dipeptidyl peptidase-4 (DPP-4) inhibitor in Japan. In our study, DS-8500a (at any dose) did not lower any of the glucose-related parameters to a greater extent than sitagliptin. However, DS-8500a treatment resulted in a notable improvement in the lipid profile, which has not been observed with sitagliptin or other DPP-4 inhibitors. Furthermore, DPP-4 inhibitors do not influence cardiovascular outcomes, but DS$8500 \mathrm{a}$ is expected to have a positive effect in this regard. On the basis of these potential effects of DS-8500a, we consider that this drug may have important clinical significance for treatment of T2DM.

Compared with sitagliptin, DS-8500a showed a weaker effect on glucose AUC after a single dose, but greater efficacy on HbA1c levels after multiple doses in a non-clinical pharmacology study [24]. However, DS-8500a did not show greater efficacy on HbA1c than sitagliptin in our 12-week clinical study. There are several possible reasons for this discrepancy between the clinical and non-clinical results. (1) Both DS-8500a and sitagliptin increased GLP-1 secretion in non-clinical studies; however, on the basis of least squares mean (LSM) vs placebo results, DS-8500a did not show such efficacy in our clinical trial, although a significant difference was observed for sitagliptin. This suggests that DS-8500a may have a weaker blood 
glucose-lowering effect than sitagliptin. (2) Sitagliptin suppressed glucagon secretion in the present study, but DS-8500a did not. Because glucagon raises plasma glucose levels by promoting glucose production in the liver, it is possible that the influence on glucagon may have accounted for the difference in blood glucose-lowering effects of sitagliptin vs DS8500a. (3) GIP levels significantly increased in the DS-8500a group compared with the placebo and sitagliptin groups in the present study (Table 2). In a non-clinical study, DS-8500a also increased GIP levels compared with vehicle (unpublished data). GIP has been suggested to be involved in insulin secretion and to contribute to plasma glucose reduction by facilitating the cellular uptake of glucose in peripheral tissues [25]. Although GIP modulation by DS-8500a is unknown, it is possible that such an effect might have influenced the difference in blood glucose lowering with sitagliptin. (4) PYY has been reported to be degraded by DPP-4 [26]. Our present findings showed a significant increase in the PYY levels in the DS-8500a 75-mg group compared with the placebo and sitagliptin groups. PYY has an appetite-suppressing effect; however, because no AEs related to appetite suppression were reported, the extent of the contribution of this effect is unknown. Nevertheless, it is possible that PYY levels might have influenced the difference in plasma glucose-lowering effects of sitagliptin.

Regarding the effect of DS-8500a on lipid profile, the present study showed that treatment with DS-8500a significantly improved total cholesterol, LDL-cholesterol, and TG levels. Improvements in lipid profiles have also been reported for other GPR119 agonists, including GSK1292263 [27] and PSN821 [28]. In the present study, the changes in HbA1c and lipids were not correlated, which suggests that they might be mediated via different mechanisms of action. The mechanism of action of the abovementioned changes in lipids is unknown. Preliminary data suggest that GPR119 agonists slow the systemic appearance of cholesterol after an oral cholesterol load in rats, or that they might promote the clearance of chylomicrons or other TG-rich lipoproteins from circulation [29]. The current hypothesis is that the mechanism of the TG-lowering effect by DS-8500a may be related to lipoprotein lipase activation by GIP. GIP, but not GLP-1, can activate lipoprotein lipase through phosphoinositide 3-kinase [30], and GIP has been shown to reduce plasma TGs in dogs [31]. Additional studies are clearly needed to evaluate the mechanism of action of GPR119 agonists on lipids.

When placing the present findings in the context of the current treatment regimen for T2DM in Japan, DS-8500a is expected to protect $\beta$-cells by enhancing first-phase insulin secretion. We believe this therapy will be effective for patients in stages of diabetes in which $\beta$-cells are not yet exhausted.

\section{Limitations}

The length of the present study ( 12 weeks of treatment with DS-8500a) may limit the significance of the results, as this is insufficient to show the long-term efficacy of DS-8500a. It will be necessary to confirm not only the long-term efficacy of DS-8500a but also the efficacy in patients in advanced stages of diabetes. Additionally, as this study was conducted in Japan, our results cannot be extrapolated to other patient populations.

\section{CONCLUSIONS}

In summary, DS-8500a was well tolerated, and all doses significantly lowered HbA1c compared with placebo. In addition, DS-8500a at 50 and $75 \mathrm{mg}$ significantly lowered FPG and 2hr-PPG compared with placebo and improved lipid parameters compared with both placebo and sitagliptin.

\section{ACKNOWLEDGEMENTS}

Some results of this study were previously presented as a poster presentation at the 53rd Annual Meeting of the European Association for 
the Study of Diabetes, Lisbon, Portugal, September 11-15, 2017.

Funding. This study was sponsored by Daiichi Sankyo Co. Ltd., Tokyo, Japan. Daiichi Sankyo Co. Ltd. also provided funding for the journal's article processing charges and open access fee.

Medical Writing Assistance. The authors would like to thank Rhonna Gurevich, PhD, of Edanz Medical Writing for medical writing support, which was funded by Daiichi Sankyo Co., Ltd.

Authorship. All named authors meet the International Committee of Medical Journal Editors (ICMJE) criteria for authorship for this manuscript, take responsibility for the integrity of the work as a whole, and have given final approval of the version to be published. All authors had full access to all of the data in this study and take complete responsibility for the integrity of the data and accuracy of the data analysis.

Disclosures. Yuichiro Yamada received honoraria from Dainippon Sumitomo Pharma Co., Ltd., Kowa Pharmaceutical Company Ltd., and Sanofi K.K.; and other fees from Dainippon Sumitomo Pharma Co., Ltd., Kowa Pharmaceutical Company Ltd., and Sanofi K.K. Yasuo Terauchi received research support from Astellas Pharma Inc., AstraZeneca K.K., Bayer Yakuhin, Ltd., Daiichi Sankyo Co., Ltd., Dainippon Sumitomo Pharma Co., Ltd., Eli Lilly Japan K.K., Kowa Pharmaceutical Company Ltd., MSD K.K., Mitsubishi Tanabe Pharma Corporation, Nippon Boehringer Ingelheim Co., Ltd., Novo Nordisk Pharma Ltd., Ono Pharmaceutical Co., Ltd., Pfizer Japan Inc., Sanwa Kagaku Kenkyusho Co., Ltd., Sanofi K.K., Shionogi \& Co., Ltd., Taisho Toyama Pharmaceutical Co., Ltd., and Takeda Pharmaceutical Co., Ltd.; and speaker's bureau fees from Astellas Pharma Inc., AstraZeneca K.K., Bayer Yakuhin, Ltd., Daiichi Sankyo Co., Ltd., Dainippon Sumitomo Pharma Co., Ltd., Eli Lilly Japan K.K., Merck Sharp \& Dohme K.K., Mitsubishi Tanabe Pharma Corporation, Nippon Boehringer Ingelheim Co.,
Ltd., Novo Nordisk Pharma Ltd., Ono Pharmaceutical Co., Ltd., Sanwa Kagaku Kenkyusho Co., Ltd., Sanofi K.K., Shionogi \& Co., Ltd., Taisho Toyama Pharmaceutical Co., Ltd., and Takeda Pharmaceutical Co., Ltd. Hirotaka Watada received research support from Novartis Pharma K.K., Eli Lilly Japan K.K., Taisho Toyama Pharmaceutical Co., Ltd., MSD K.K., Astellas Pharma Inc., AstraZeneca K.K., Ono Pharmaceutical Co., Ltd., Kyowa Hakko Kirin Co., Ltd., Sanofi K.K., Daiichi Sankyo Co., Ltd., Dainippon Sumitomo Pharma Co., Ltd., Takeda Pharmaceutical Co., Ltd., Mitsubishi Tanabe Pharma Corporation, Terumo Corporation, Nippon Boehringer Ingelheim Co., Ltd., Novo Nordisk Pharma Ltd., Pfizer Japan Inc., Benefit One Health care Inc., Mochida Pharmaceutical Co., Ltd., and Nitto Boseki Co., Ltd.; and speaker's bureau fees from Astellas Pharma Inc., AstraZeneca K.K., Kowa Pharmaceutical Co., Ltd., Sanofi K.K., Takeda Pharmaceutical Co., Ltd., Mitsubishi Tanabe Pharma Corporation, Novartis Pharma K.K., Novo Nordisk Pharma Ltd., Nippon Boehringer Ingelheim Co., Ltd., MSD K.K., and Dainippon Sumitomo Pharma Co., Ltd. Yasuhiko Nakatsuka is an employee of Daiichi Sankyo. Kazuhito Shiosakai is an employee of Daiichi Sankyo. Takuo Washio is an employee of Daiichi Sankyo. Takashi Taguchi is an employee of Daiichi Sankyo.

Compliance with Ethics Guidelines. This study received approval by the institutional review board at each site (see Supplementary Table S1 for a list of sites). This study conformed with the Helsinki Declaration of 1964, as revised in 2013, concerning human and animal rights, and Springer's policy concerning informed consent has been followed. All procedures followed were in accordance with the ethical standards of the responsible committee on human experimentation (institutional and national), and with the Helsinki Declaration of 1964 , as revised in 2013. Informed consent was obtained from all patients for being included in the study.

Data Availability. The datasets for and/or analyzed during the current study are available 
from the corresponding author on reasonable request.

Open Access. This article is distributed under the terms of the Creative Commons Attribution-NonCommercial 4.0 International License (http://creativecommons.org/licenses/ by-nc/4.0/), which permits any noncommercial use, distribution, and reproduction in any medium, provided you give appropriate credit to the original author(s) and the source, provide a link to the Creative Commons license, and indicate if changes were made.

\section{REFERENCES}

1. Mayo Clinic. Type 2 diabetes; 2017. http://www. mayoclinic.org/diseases-conditions/type-2diabetes/home/ovc-20169860. Accessed 8 June 2017.

2. International Diabetes Federation. IDF Diabetes Atlas, sixth edition. Brussels: IDF; 2013.

3. Ministry of Health, Labour and Welfare. Summary results of the National Health and Nutrition Survey Japan 2007. Tokyo: Ministry of Health, Labour and Welfare; pp. 3-29.

4. NCD Risk Factor Collaboration. Worldwide trends in diabetes since 1980: a pooled analysis of 751 population-based studies with 4.4 million participants. Lancet. 2016;387(10027):1513-30.

5. Mayo Clinic. Diabetes treatment: medication for type 2 diabetes; 2014. http://www.mayoclinic.org/ diseases-conditions/type-2-diabetes/in-depth/ diabetes-treatment/art-20051004. Accessed 1 Sept 2017.

6. Soga T, Ohishi T, Matsui T, et al. Lysophosphatidylcholine enhances glucose-dependent insulin secretion via an orphan G-protein-coupled receptor. Biochem Biophys Res Commun. 2005;326:744-51 (Erratum in: Biochem Biophys Res Commun. 2005; 329:417).

7. Chu ZL, Carroll C, Alfonso J, et al. A role for intestinal endocrine cell-expressed $\mathrm{G}$ protein-coupled receptor 119 in glycemic control by enhancing glucagon-like peptide-1 and glucose-dependent insulinotropic peptide release. Endocrinology. 2008;149:2038-47.

8. Chu ZL, Jones RM, He $\mathrm{H}$, et al. A role for $\beta$-cellexpressed G protein-coupled receptor 119 in glycemic control by enhancing glucose-dependent insulin release. Endocrinology. 2007;148:2601-9.

9. Yoshida S, Ohishi $\mathrm{T}$, Matsui $\mathrm{T}$, et al. The role of small molecule GPR119 agonist, AS1535907, in glucose-stimulated insulin secretion and pancreatic $\beta$-cell function. Diabetes Obes Metab. 2011;13:34-41.

10. Matsumoto K, Yoshitomi T, Takahashi K, et al. DS$8500 a$, a novel orally available GPR119 agonist improves glucose tolerance in type 2 diabetic rats. Diabetes. 2016;65(Suppl 1):1124-P.

11. Kato M, Furuie H, Tsuruta F. Safety and pharmacokinetics of DS-8500a, a novel GPR119 agonist, after multiple oral doses in healthy Japanese males. Diabetes. 2016;65(Suppl 1):1187-P.

12. Inagaki N, Chou HS, Tsukiyama $S$, et al. Glucoselowering effects and safety of DS-8500a, a G protein-coupled receptor 119 agonist, in Japanese patients with type 2 diabetes: results of a randomized, double-blind, placebo-controlled, parallelgroup, multicenter, phase II study. BMJ Open Diabetes Res Care. 2017. https://doi.org/10.1136/ bmjdrc-2017-000424.

13. International Hypoglycaemia Study Group. Glucose concentrations of less than $3.0 \mathrm{mmol} / \mathrm{L}(54 \mathrm{mg} / \mathrm{dL})$ should be reported in clinical trials: a joint position statement of the American Diabetes Association and the European Association for the Study of Diabetes. Diabetes Care. 2017;40:155-7.

14. Pharmaceuticals and Medical Devices Agency. Guideline on clinical evaluation method of hypoglycemic drugs (revision). 2010. https://www. pmda.go.jp/files/000208194.pdf. Accessed 5 Oct 2017.

15. Nonaka K, Tsubouchi $\mathrm{H}$, Okuyama K, Fukao $\mathrm{Y}$, Johnson-Levonas AO, Amatruda JM. Effects of once-daily sitagliptin on 24-h glucose control following 4 weeks of treatment in Japanese patients with type 2 diabetes mellitus. Horm Metab Res. 2009;41:232-7.

16. Katz LB, Gambale JJ, Rothenberg PL, et al. Effects of JNJ-38431055, a novel GPR119 receptor agonist, in randomized, double-blind, placebo-controlled studies in subjects with type 2 diabetes. Diabetes Obes Metab. 2012;14:709-16.

17. Nunez DJ, Bush MA, Collins DA, et al. Gut hormone pharmacology of a novel GPR119 agonist (GSK1292263), metformin, and sitagliptin in type 2 diabetes mellitus: results from two randomized studies. PLoS One. 2014;9:e92494.

18. McClure KF, Darout E, Guimarães CR, et al. Activation of the G-protein-coupled receptor 119: a 
conformation-based hypothesis for understanding agonist response. J Med Chem. 2011;54:1948-52.

19. Yabe D, Seino Y, Fukushima M, et al. Beta cell dysfunction versus insulin resistance in the pathogenesis of type 2 diabetes in East Asians. Curr Diab Rep. 2015;15:602.

20. Kodama K, Tojjar D, Yamada S, et al. Ethnic differences in the relationship between insulin sensitivity and insulin response: a systematic review and meta-analysis. Diabetes Care. 2013;36:1789-96.

21. Seino Y, Kuwata H, Yabe D. Incretin-based drugs for type 2 diabetes: focus on East Asian perspectives. J Diabetes Investig. 2016;7(Suppl 1):102-9.

22. Kim YG, Hahn S, Oh TJ, et al. Differences in the HbA1c-lowering efficacy of glucagon-like peptide-1 analogues between Asians and non-Asians: a systematic review and meta-analysis. Diabetes Obes Metab. 2014;16:900-9.

23. Watada $\mathrm{H}$, Shiramoto $\mathrm{M}$, Irie $\mathrm{S}$ et al. DS-8500a, a GPR-119 agonist, enhanced insulin secretory capacity in a hyperglycemic clamp study in patients with type 2 diabetes mellitus. Diabetes. 2017;66(Suppl 1A):154-LB.

24. Yoshitomi T, Matsumoto K, Tanaka N, Takahashi K. DS-8500a, a novel GPR119 agonist preserves pancreatic $\beta$-cell function and prevents glycohemoglobin increase compared with sitagliptin in type 2 diabetic mice. Diabetes. 2016;65(Suppl 1A):1192-P.

25. Kim W, Egan JM. The role of incretins in glucose homeostasis and diabetes treatment. Pharmacol Rev. 2008;60:470-512.
26. Aaboe K, Knop FK, Vilsbøll T, et al. Twelve weeks treatment with the DPP-4 inhibitor, sitagliptin, prevents degradation of peptide YY and improves glucose and non-glucose induced insulin secretion in patients with type 2 diabetes mellitus. Diabetes Obes Metab. 2010;12:323-33.

27. A study in type 2 diabetic subjects of single and multiple doses of orally administered GSK1292263 to investigate the safety, tolerability, pharmacokinetics and pharmacodynamics of the compound (study GPR111598). 2012. http://www.gskclinicalstudyregister.com/study/111598. Accessed Nov 2017.

28. Goodman ML, Dow J, van Vliet AA, Pleszko A, Lockton JA. Orally administered GPR119 agonist PSN821 shows clinically significant glucose lowering and other potential cardiometabolic benefits in patients with T2DM. Diabetologia. 2011;54:Abstract OP32.

29. Brown KK, Shadoan MK, Croom DK, et al. Activation of GPR119 reduces the appearance of labeled cholesterol in an oral fat tolerance test. Diabetes. 2012;61(Suppl 1):A160 (631-P).

30. Kim SJ, Nian C, McIntosh CH. Activation of lipoprotein lipase by glucose-dependent insulinotropic polypeptide in adipocytes. A role for a protein kinase B, LKB1, and AMP-activated protein kinase cascade. J Biol Chem. 2007;282:8557-67.

31. Wasada T, McCorkle K, Harris V, Kawai K, Howard B, Unger RH. Effect of gastric inhibitory polypeptide on plasma levels of chylomicron triglycerides in dogs. J Clin Invest. 1981;68:1106-7. 